\title{
Surfactant-based Transdermal System for Fluconazole Skin Delivery
}

Hilris Rocha e Silva', Gabriela Marielli da Luz', Cínthia Yuka Satake'1, Bruna Carolina Correa', Victor Hugo Vitorino Sarmento², Georgino Honorato de Oliveira' ${ }^{1}$, Flávia Chiva Carvalho ${ }^{1,3}$, Marlus Chorilli' ${ }^{1}$ and Maria Palmira Daflon Gremião ${ }^{1 *}$

${ }^{1}$ School of Pharmaceutical Sciences, Universidade Estadual Paulista UNESP, Araraquara, SP, Brazil

${ }^{2}$ Department of Chemistry, Federal University of Sergipe, Av. Vereador Olimpio Grande $\mathrm{s} / \mathrm{n}$, Centro - Itabaiana-SE, Brazil

${ }^{3}$ Department of Food and Medicines, Federal University of Alfenas, UNIFAL-MG, Alfenas, MG, Brazil

\begin{abstract}
The development of a controlled-release dosage form of antifungals is of crucial importance in view of the side-effects of conventional oral and intravenous treatments of Sporotrichosis. In this study, systems composed of polyoxypropylene (5) polyoxyethylene (20) cetyl alcohol (PPG-5-CETETH-20) as a surfactant, oleic acid as an oil phase, and water were developed as a possible fluconazole transdermal drug delivery system. The systems were characterised by polarised light microscopy (PLM), SAXS, and rheological analysis, followed by cellular and histological analyses, in vitro release assays, and ex vivo skin permeation and retention studies using porcine ear tissue and a Franz diffusion cell. PLM and SAXS results indicated that the mixtures of surfactant, oil and water formed micellar and lamellar phases. The incorporation of fluconazole in these systems was greater than in water and conventional dosage forms. Micellar systems behave as Newtonian fluids, being more viscous than elastic in rheological analysis, and lamellar phases behave as pseudoplastic fluids with high elastic moduli. In vitro and in vivo biological assays showed that the formulations did not affect normal cell macrophages and did not promote skin irritation. The release profile indicated that fluconazole could be released in a controlled manner. It was found that the systems enhanced drug permeation and skin retention by changing only the composition of the components in the formulations. Therefore, PPG-5-CETETH-20based systems have great potential as transdermal systems with different structural and rheological characteristics for Sporotrichosis treatment using antifungal drugs.
\end{abstract}

Keywords: PPG-5-CETETH-20; Transdermal drug delivery system; Sporotrichosis treatment; Fluconazole; Phase behaviour; Surfactant systems; Liquid crystals

\section{Introduction}

Sporotrichosis is the most prevalent subcutaneous mycosis and is caused by a dimorphic fungus, Sporothrix schenckii, which has been isolated from soil, decaying vegetation, plants, timber, hay, and moss. The disease usually begins after trauma that inoculates the microorganism into the skin and subcutaneous tissue [1]. Sporotrichosis has a worldwide distribution, although it is more frequent in tropical and subtropical areas with warm and humid climates. In South America, the estimated annual incidence is 48 to 60 cases per 100,000 population [2].

Several modalities have been used to treat cutaneous Sporotrichosis, such as local hyperthermia, cryotherapy, and antifungals (potassium iodide, itraconazol, amphotericin B, terbina and fluconazole), which are mainly administered by intravenous or oral route for a systemic effect $[2,3]$. However, most of them cause side-effects, including gastric irritation, diarrhoea, nausea, vomiting and stomach pain, hypothyroidism, fever, chills, headache, impairment of renal function, and anaemia [3].

One strategy for avoiding gastrointestinal side effects is transdermal delivery [4]. There are several instances in which the most convenient drug intake method (the oral route) is not feasible, and thus, alternative routes must be investigated [5]. Although the intravenous route may avoid gastrointestinal side-effects, it is invasive, and few patients are compliant. These disadvantages have encouraged research on alternative strategies, and the transdermal route has great potential for optional drug delivery [5]. Transdermal drug delivery not only avoids the digestive system, it also effectively reduces the applied dose [4].

Drug diffusion through the skin may limit the drug action of transdermally delivered drugs. Surfactant systems such as microemulsions and liquid crystals have great potential as topical vehicles. Surfactants are able to stabilise mixtures of components with different polarities, thus creating additional regions for hydrophilic and lipophilic solubilisation of drugs. The drug can be protected against the dissolution medium due to the interfacial membrane, which is composed of the surfactant layer that the drug must pass through to be released. In this manner, surfactant systems also confer reservoir properties. The phase behaviour of surfactants enable the formation of microemulsions and liquid crystals, which is extremely attractive for nanotechnology because these structures are organised on the nanometric scale. In addition to the advantage of increased stability, their production can be scaled up easily. Varying the composition and proportions of surfactant, oil, and aqueous phase, it is possible to control the viscosity and texture of the systems above, which could lead to the immobilisation or localisation of the formulation at specific sites in the body and routes of administration. Therefore, surfactant systems have great potential as transdermal nanostructured drug delivery systems [6].

Recently, our research group has demonstrated that the surfactant polyoxypropylene (5) polyoxyethylene (20) cetyl alcohol (PPG-5CETETH-20) is capable of stabilising systems that form nanostructures, including microemulsions and several liquid crystalline mesophases,

*Corresponding author: Maria Palmira Daflon Gremião, Universidade Estadual Paulista UNESP, School of Pharmaceutical Sciences, Drugs and medicines Department, Rodovia Araraquara-Jaú, Km 01, 14801-902, Araraquara, SP, Brazil, Tel: 55-16-33016975; Fax: 55-16-33220073; E-mail: pgremiao@fcfar.unesp.br

Received August 16, 2014; Accepted October 06, 2014; Published October 14 2014

Citation: Silva HR, Luz GM, Satake CY, Correa BC, Sarmento VHV, et al. (2014) Surfactant-based Transdermal System for Fluconazole Skin Delivery. J Nanomed Nanotechnol 5: 231. doi: 10.4172/2157-7439.1000231

Copyright: $\odot 2014$ Silva HR, et al. This is an open-access article distributed under the terms of the Creative Commons Attribution License, which permits unrestricted use, distribution, and reproduction in any medium, provided the original author and source are credited. 
such as lamellar, hexagonal, and cubic phases, depending on the proportion of the components and the oily phases [6]. This capacity has been exploited as a technological platform to develop new drug delivery systems. Zidovudine (AZT)-loaded PPG-5-CETETH-20 systems were shown to enhance the permeation of the drug across the nasal mucosa in ex vivo tests employing a diffusion chamber and excised porcine nasal mucosa. The in vivo results of the nasal administration of AZT-loaded PPG-5-CETETH-20 systems in rats revealed fast absorption $\left(\mathrm{C}_{\max }=6.67\right.$ $\min )$ compared to commercial oral formulations $\left(\mathrm{C}_{\max }=0.5-1.5 \mathrm{~h}\right)$ [7]. Another study of a Propolis microparticle-loaded PPG-5-CETETH-20based system designed for periodontal pocket administration showed a prolonged release (more than 7 days) [8].

Therefore, the objective of this study was to develop and characterise PPG-5-CETETH-20-based systems for transdermal administration of antifungals. Fluconazole was used as the drug model because it is a triazole antifungal agent with broad-spectrum activity. It is effective for deep seated mycosis caused by Candida, Cryptococcus spp., and others and also for superficial infections caused by dermatophytes such as Microsporum and Trichophyton spp [9].

The systems were characterised by polarised light microscopy (PLM), small-angle X-ray scattering (SAXS), and rheological measurements. The safety was also tested using an in vitro cellular assay and in vivo histopathological analysis. In addition, ex vitro studies were conducted to examine the extent of the binding of fluconazole-loaded PPG-5-CETETH-20-based systems to the skin and their transdermal behaviour by in vitro permeation experiments using porcine ears and a Franz diffusion cell.

\section{Methodology}

\section{Materials}

The surfactant PPG-5-CETETH-20 is available commercially as Procetyl AWS (Croda, Campinas, Brazil). Oleic acid was acquired from Synth (Diadema, Brazil), and fluconazole was acquired from Purifarma, São Paulo, Brazil. High-purity water was prepared with a Millipore Milli-Q plus purification system.

\section{Preparation of the formulations}

The formulations were prepared by manual stirring using PPG-5CETETH-20 as a surfactant, oleic acid as an oily phase, and water as an aqueous phase in a beaker. The proportions of the constituents are indicated in Table 1 . The formulations were prepared $24 \mathrm{~h}$ before the experiments and left at room temperature to allow the system to reach complete equilibrium. Fluconazole was incorporated by dissolving the drug powder directly into the systems.

\section{Evaluation of the fluconazole solubility in the formulations}

The solubility of fluconazole in the formulations was investigated by adding crescent specific amounts of the drug powder $(0.01 \% \mathrm{w} / \mathrm{w})$ to $2 \mathrm{~g}$ of each system and to their isolated components. The mixtures were manually homogenised using a glass rod and maintained at room temperature for $24 \mathrm{~h}$. This procedure was repeated until precipitation was observed. Loaded samples were named AO1F, AO2F, AO3F, and $\mathrm{AO} 4 \mathrm{~F}$.

Fluconazole was analysed by HPLC, with the UV detector set at $210 \mathrm{~nm}$ (Varian Pro Star 330). A reverse-phase Varian Chromsep C18 column $(250 \times 4.6 \mathrm{~mm}$ i.d., $5 \mu \mathrm{m}$ pore size $)$ was used. The mobile phase was a mixture of methanol/water $(1: 1 \mathrm{v} / \mathrm{v})$, the flow rate was set at $1 \mathrm{~mL} / \mathrm{min}$, and the injection volume was $20 \mu \mathrm{L}$. A calibration curve was constructed by preparing working solutions of fluconazole in the mobile phase at concentrations ranging from 20 to $400 \mu \mathrm{g} / \mathrm{mL}$.

Samples were prepared by diluting the formulation into the mobile phase to obtain a drug concentration of $150 \mu \mathrm{g} / \mathrm{mL}$. The dilution was cleaned up by solid phase extraction (SPE) using $500 \mathrm{mg}$ per $3 \mathrm{~mL} \mathrm{C18}$ cartridge. The SPE procedure included conditioning the samples with 1 $\mathrm{mL}$ of the mobile phase. The spiked samples $(2.5 \mathrm{~mL})$ were then loaded into the conditioned cartridge, and the analytes were collected, filtered through $0.45 \mu \mathrm{m}$-pore membranes, and injected into the HPLC system.

\section{Studies of phase behaviour by polarised light microscopy (PLM) and small-angle X-ray scattering (SAXS)}

PLM was performed in an optical Jenamed 2, Carl Zeiss (Jena, Germany) microscope. The isotropic or anisotropic behaviour of the samples was observed, and pictures were taken at a $20,000 \times$ magnification. Samples were prepared by placing a drop of formulation between a cover slip and a glass slide. They were then examined under polarised light at room temperature.

SAXS data were collected at the Synchrotron SAXS beam line of the National Laboratory of Synchrotron Light (LNLS, Campinas, Brazil), equipped with an asymmetrically cut and bent Si $\left(\begin{array}{lll}1 & 1 & 1\end{array}\right)$ monochromator $(\lambda=1.608 \AA)$ that yields a horizontally focused beam. A vertical position-sensitive X-ray detector and a multichannel analyser were used to record the SAXS intensity, $I(q)$, as a function of the modulus of the scattering vector $\mathrm{q}(\mathrm{q}=(4 \pi / \lambda) \sin (\varepsilon / 2))$, with $\varepsilon$ being the scattering angle. The samples were placed in a cell at $25^{\circ} \mathrm{C}$. The parasitic scattering produced by the slits was subtracted from the total scattering intensity.

\section{Rheological studies}

The rheological measurements were taken with a controlled stress Rheostress RS1 rheometer (Haake, Karlsruhe, Germany) and analysed with Rheowin 3.5 software. Plate-plate geometry was used with a gap of $200 \mu \mathrm{m}$ between plates $35 \mathrm{~mm}$ in diameter. Samples were carefully applied to the lower plate, ensuring that sample shearing was minimised, and were allowed to equilibrate for at least $3 \mathrm{~min}$ prior to analysis.

The flow properties were determined with shear rates within the range of $0.01-100 \mathrm{~s}^{-1}$, which was chosen on the basis of the strength of resistance to the applied stresses. The rheological measurements were

\begin{tabular}{|c|c|c|c|}
\hline & \multicolumn{2}{|c|}{ Fluconazole } \\
\cline { 2 - 4 } & Oleic acid & PPG-5-CETETH-20 & Water \\
\hline AO1 & 20 & 40 & 40 \\
\hline AO2 & 30 & 40 & 30 \\
\hline AO3 & 40 & 40 & $24.43 \pm 0.99$ \\
\hline AO4 & 50 & 40 & $26.34 \pm 1.00$ \\
\hline $\begin{array}{c}\text { Fluconazole } \\
\text { solubility (mg/g) }\end{array}$ & 4 & 15 & $29.78 \pm 1.68$ \\
& & \\
\end{tabular}

Table 1: Composition, phase behaviour, and fluconazole solubility of the studied formulations 
performed on both the up and down curves. The flow curves were fitted to a power law model using the program Origin 7.0. All rheological determinations were carried out at $25 \pm 0.25^{\circ} \mathrm{C}$

Oscillatory analysis of each sample was performed after determination of its linear viscoelastic region at $25^{\circ} \mathrm{C}$, where stress was directly proportional to strain and the storage modulus remained constant. Frequency sweep analysis was performed over the frequency range of $0.1-10 \mathrm{~Hz}$ at a constant stress of $1 \mathrm{~Pa}$. The systematic error in the frequency of the rheometer was approximately $0.01 \mathrm{~Hz}$.

\section{Safety evaluation of the formulations}

In vitro unspecific cytotoxicity: Mammal cytotoxicity of the formulations was studied in vitro using J-774 mouse macrophages as the cellular model. Cells were seeded at a density of $2.5-10.0 \times 10^{5}$ cells/ well in 96-well flat bottom microplates (Nunclon) and exposed for 48 $\mathrm{h}$ to different doses of the formulations and free-drug (18.6, 10, 5 and $1 \mu \mathrm{M}$ ) for control. After treatment, the compounds were removed, and the cells washed once with PBS. Cell viability was then colorimetrically assessed by measuring the mitochondrial-dependent reduction of MTT to formazan. For this purpose, the cells and MTT $(0.4 \mathrm{mg} / \mathrm{mL})$ were incubated in air at $37^{\circ} \mathrm{C}$ for $3 \mathrm{~h}$. After the incubation period, the supernatant was removed, and formazan crystals were dissolved with DMSO $(180 \mu \mathrm{L})$. The plates were shaken for $10 \mathrm{~min}$, and the optical densities were measured at $560 \mathrm{~nm}$ in a multiwell spectrophotometer. Each concentration was assayed three times, and six additional controls (cells in medium) were used in each test. Data were exhibited in per cent of cellular viability.

\section{In vivo evaluation}

Animals: During the experiment, the animals were maintained in accordance with the guidelines established by Olfert et al. [10] and the Statement of Principles adopted by the Federation of American Societies for Experimental Biology (FASEB) Board. The animals had free access to food and water. Twenty-four hours before the start of treatment, areas of $4 \mathrm{~cm} \times 5 \mathrm{~cm}$ were outlined on the back of each animal. The raw materials and formulation AO3F were tested. Each experimental group consisted of five rats. The animals were given the following treatments: oleic acid (OA), PPG-5-CETETH-20 (PROC), formulation $\mathrm{AO} 3 \mathrm{~F}$, benzalkonium chloride $(\mathrm{BC})$ solution $15 \%$ (positive control, C+), and propanol (negative control, C-). All areas, except the control area, were subjected to a daily treatment with the indicated formulations for 21 days, always at the same time, accompanied by frictional circular massage.

Histological procedures: The animals were anesthetised with xylazine hydrochloride / ketamine hydrochloride and sacrificed in a $\mathrm{CO}_{2}$ chamber, after which the areas of treated skin were removed, fixed in Bouin's solution for 48 hours, rinsed, and treated routinely for embedding in paraffin (Histosec, Merck). Five non-serial histological sections $(5 \mu \mathrm{m})$ were cut for each slide, with three slides per treatment area. The sections were stained in hematoxylin and eosin (HE) for histomorphometric and histopathological analyses.

Histomorphometric analysis was performed by capturing images of random cuts through an optical microscope Leica DMZ 2000 connected to a computer with the software Motic Images Advanced. Thirty areas were randomly chosen, and the thickness of the epidermis and dermis for each treatment area was measured. From these measurements, a correction for the micrometre scale was calculated for an objective of 20x, which yielded the mean thickness and standard deviation for each experimental group.
Histopathological analysis consisted of counting the number of leukocytes and fibroblasts using a Leica DMZ 2000 optical microscope connected to a computer with the software Motic Images Advanced 3.2. For each experimental group, three slides with 5 nonserial sections per slide were obtained. Six areas of $250 \mu^{2}$ of skin per experimental group, always in the region of the papillary dermis, were measured. Therefore, each treatment comprised a total area of $30,000 \mu \mathrm{m}^{2}$ per animal. From these results, the mean and standard deviation for each cell type was determined for each treatment.

Statistical analysis was performed using the Kruskal-Wallis statistical test, followed by the Wilcoxon-Mann-Whitney test with Bioestat version 5.0. The level of significance adopted was $5 \%(\mathrm{p}<0.05)$.

\section{Proof of concept of the formulations by drug release, drug} skin permeation, and drug skin retention studies

These studies comprise drug release, skin permeation, and skin retention assays [11]. The loaded-formulations and loaded-oleic acid containing fluconazole were tested at $10 \mathrm{mg} / \mathrm{mL}$.

In the drug release study, a $45 \mu \mathrm{m}$-pore size cellulose acetate membrane was used to separate the formulations from the release medium. For drug permeation and retention studies, porcine ear skin obtained from healthy 6-month-old Brazilian pigs of a local slaughterhouse and prepared for the test as described elsewhere [12] were used. The ears were cleaned with water $\left(25 \pm 0.5^{\circ} \mathrm{C}\right)$, and the ears with injury were discarded. The undamaged skins were removed from the cartilage with a scalpel, and a $500 \mu \mathrm{m}$ thick layer stratum corneum and epidermis were separated from the adipose tissue with a dermatome (Nouvag TCM 300, Goldach, USA). The prepared skins were frozen at $-20^{\circ} \mathrm{C}$ and stored no longer than 4 weeks. On the day of the experiment, the skin was thawed in physiological saline solution containing $0.9 \%(\mathrm{w} / \mathrm{v}) \mathrm{NaCl}$ (Merck, Germany) at $25 \pm 0.5^{\circ} \mathrm{C}$ for 30 min; then, its hair was cut with a scissor.

The cellulose acetate membranes or skin pieces were placed between the donor and receptor chambers of a Franz diffusion cell (Microette Plus, Hanson Research, Chatsworth, EUA) equipped with diffusion cells with an exposition area of $1.77 \mathrm{~cm}^{2}$ for formulation deposition. The receptor chambers of the cells $(7 \mathrm{~mL})$ were filled with $10 \mathrm{mM}$ monobasic buffer ( $\mathrm{pH} 7.4$ ) stirred at $300 \mathrm{rpm}$. The samples (400 $\mathrm{mg}$ ) were dropped on the donor chambers in contact with the receptor chambers. The total test time was $24 \mathrm{~h}$, and $2 \mathrm{~mL}$ of the receptor fluid was collected and replaced by an equal volume of fresh receptor solution. The assays were carried out on six replicates for each sample.

Fluconazole was quantified by HPLC using the same conditions described for the fluconazole solubility study. A calibration curve was constructed by preparing working solutions of fluconazole in $10 \mathrm{mM}$ monobasic phosphate buffer ( $\mathrm{pH}$ 7.4) at concentrations ranging from 1 to $250 \mu \mathrm{g} / \mathrm{mL}$. Samples of collected receptor fluid were filtered and injected into the HPLC system.

The results are represented by a graph of the cumulative mass of the drug that passes through a unit area of the membrane or skin versus time, calculated according to equation 1 :

$$
Q=C_{t} \times V_{r}+\sum V_{c} \times C_{c}
$$

where $\mathrm{Q}\left(\mu / \mathrm{cm}^{2}\right)$ is the total amount of drug in the receptor fluid up to time $\mathrm{t}, \mathrm{C}_{\mathrm{t}}\left(\mu \mathrm{g} / \mathrm{mL} \mathrm{cm}^{2}\right)$ is the concentration measured at time $\mathrm{t}$, $\mathrm{V}_{\mathrm{r}}(\mathrm{mL})$ is the volume of receptor solution $(7 \mathrm{~mL}), \mathrm{C}_{c}\left(\mu \mathrm{g} / \mathrm{mL} . \mathrm{cm}^{2}\right)$ is the concentration at the previous sampling, and $\mathrm{V}^{\mathrm{c}}(\mathrm{mL})$ is the volume sampled. 


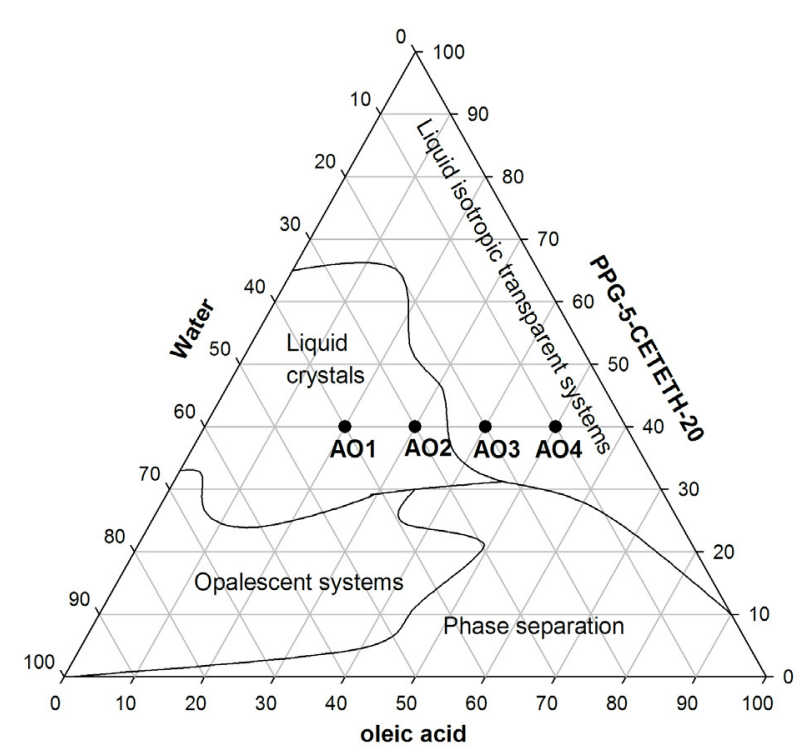

Figure 1: Ternary phase diagram of PPG-5-CETETH 20, oleic acid, and water, obtained from previous studies by Carvalho et al. [6].

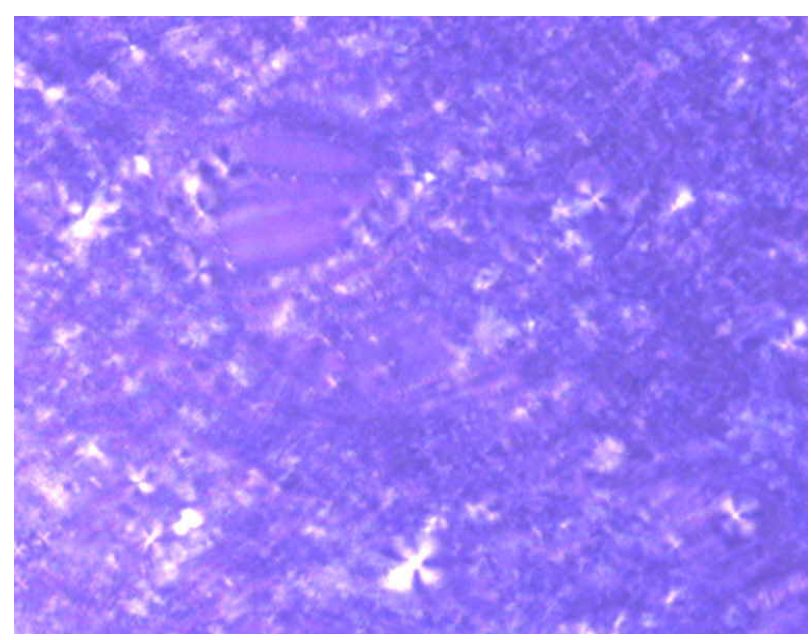

Figure 2: Representative photomicrograph obtained using PLM that shows the streaks of the Maltese crosses visualised in $\mathrm{AO} 3$ and $\mathrm{AO} 4$

For retention studies, the tape stripping method [13-15] was performed. The skins derived after $6 \mathrm{~h}$ and $12 \mathrm{~h}$ of permeation assay were cleaned using soft paper. The stratum corneum was removed from sixteen tapes (Scotch 750,3M), and retained fluconazole was evaluated by solvent extraction. The tapes were placed in an assay tube containing $5 \mathrm{~mL}$ of methanol and vortexed for $2 \mathrm{~min}$. Then, the sample was immersed in an ultrasonic bath for $30 \mathrm{~min}$. The solvent was filtered through a $0.45 \mu \mathrm{m}$ membrane and injected into the HPLC system. The remaining skin was cut into small pieces, placed in a tube containing 5 $\mathrm{mL}$ of methanol, and tested according to the same procedure.

\section{Results and Discussion}

\section{Preparation of the formulations}

The composition of the formulations was chosen based on previous studies in which the ternary phase diagram of oleic acid, PPG-5-
CETETH-20 and water mixtures was obtained (Figure 1). For this study, thermodynamically stable systems, characterised by their spontaneous formation, transparency and high stability, were selected. Samples AO1 and $\mathrm{AO} 2$ correspond to semisolid transparent systems, and $\mathrm{AO} 3$ and $\mathrm{AO} 4$ correspond to liquid and transparent systems. Their compositions are given in Table 1. For AO1-4, the concentration of the surfactant was fixed at $40 \%(\mathrm{w} / \mathrm{w})$, whereas the other component concentrations were varied. These compositions were chosen to avoid the translucent dispersion and phase separation areas.

\section{Evaluation of fluconazole solubility in the formulations}

The incorporation of fluconazole into the individual oil, surfactant, water, and formulations was measured, and the results are displayed in Table 1. The formulations incorporated more drug than water and surfactant but less drug than oleic acid by itself. These results confirm the lipophilic character of fluconazole. It is worth highlighting that the formulations incorporated more fluconazole than conventional topical pharmaceutical formulations $(10 \mathrm{mg} / \mathrm{g})$ due to the mixture of components with different polarities, namely, oil and water, in the presence of a surfactant that forms aggregates and allows additional regions of drug solubilisation.

\section{Phase behaviour study by PLM and SAXS}

The semisolid samples (AO1 and $\mathrm{AO} 2)$ were anisotropic under polarised light, which is characteristic of liquid crystals that are birefringent as a result of their molecular ordering. The images obtained by PLM for AO1 and AO2 are shown in Figure 2, in which Maltese crosses characteristic of lamellar phases can be seen.

The transparent liquid systems investigated ( $\mathrm{AO} 3$ and $\mathrm{AO} 4)$ showed a dark background under PLM, and thus, they are isotropic systems that are characteristic of micellar systems, microemulsions, or cubic phases. Cubic phases are well known by their high stiffness, and AO3AO4 flowed easily. Therefore, they may not be organised structurally as a mesophase.

The phase behaviour of the systems was confirmed by the SAXS measurements. The data that were collected from the scattering profiles were plotted as intensity, $(I)$, versus the scattering vector module, $\mathrm{q}(\AA$ $\left.{ }^{1}\right)$. The resulting curves are shown in Figure 3.

Surfactants can form different aggregate types, which can be identified by the peak positions on the scattering vector axis. From the scattering profile of loaded and unloaded $\mathrm{AO} 1-\mathrm{AO} 2$ formulations, two peaks were observed, possibly because of a degree of structural organisation. The ratio between the first and second peak is $2: 1$, which is characteristic of lamellar liquid crystals [16]. This result correlates with the PLM experiments because Malta crosses were visualised for the $\mathrm{AO} 1$ and $\mathrm{AO} 2$ systems. For the $\mathrm{AO} 3$ and $\mathrm{AO} 4$ formulations, a maximum intensity value of $\mathrm{q} \neq 0$ followed by a long tail was observed, which is associated with short-range $3 \mathrm{D}$ spatial correlation and may be characteristic of micellar structures [17].

\section{Rheological studies}

The flow properties of these systems are displayed graphically in Figure 4 , in which the shear stress is plotted versus the shear rate. In the continuous shear rheometry assay, both unloaded and loaded AO3AO4 systems exhibited Newtonian behaviour. This result was expected because the samples consisted of isotropic liquid systems. The curves of AO1-AO2 showed a nonlinear relationship between shear stress and shear rate, thus exhibiting a non-Newtonian flow characteristic. These results are in accord with literature data showing that liquid crystalline phases are found to exhibit shear-thinning behaviour. 
Citation: Silva HR, Luz GM, Satake CY, Correa BC, Sarmento VHV, et al. (2014) Surfactant-based Transdermal System for Fluconazole Skin Delivery. J Nanomed Nanotechnol 5: 231. doi: 10.4172/2157-7439.1000231
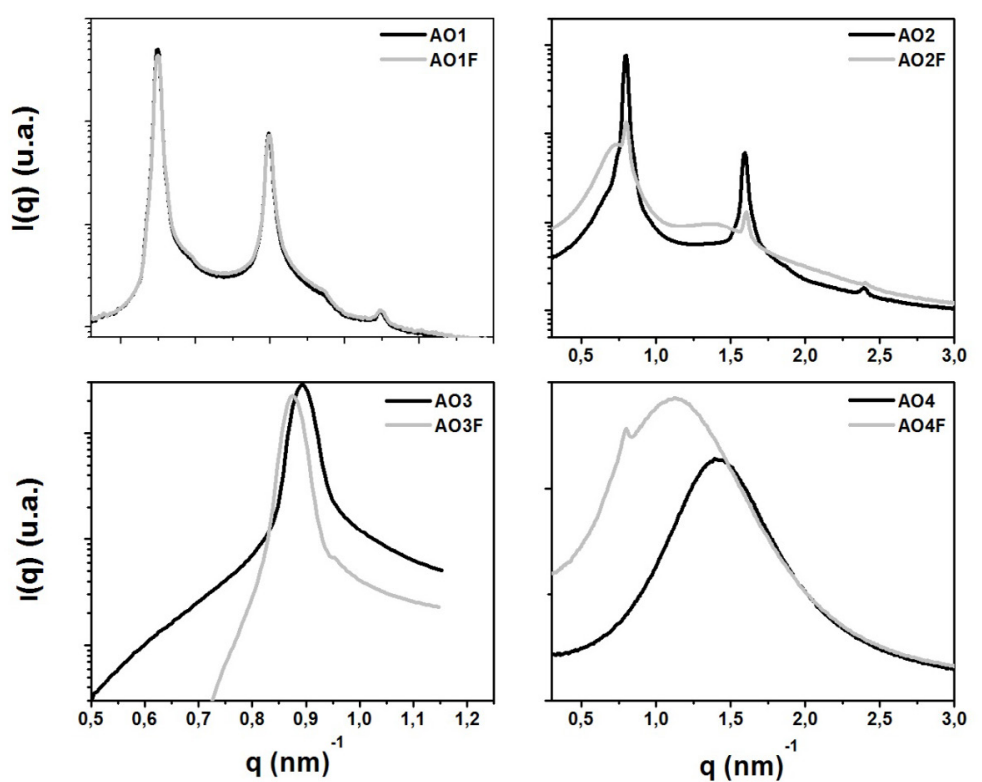

Figure 3: SAXS patterns of samples of fluconazole-loaded (AO1F-AO4F) and unloaded (AO1-AO4) formulations.
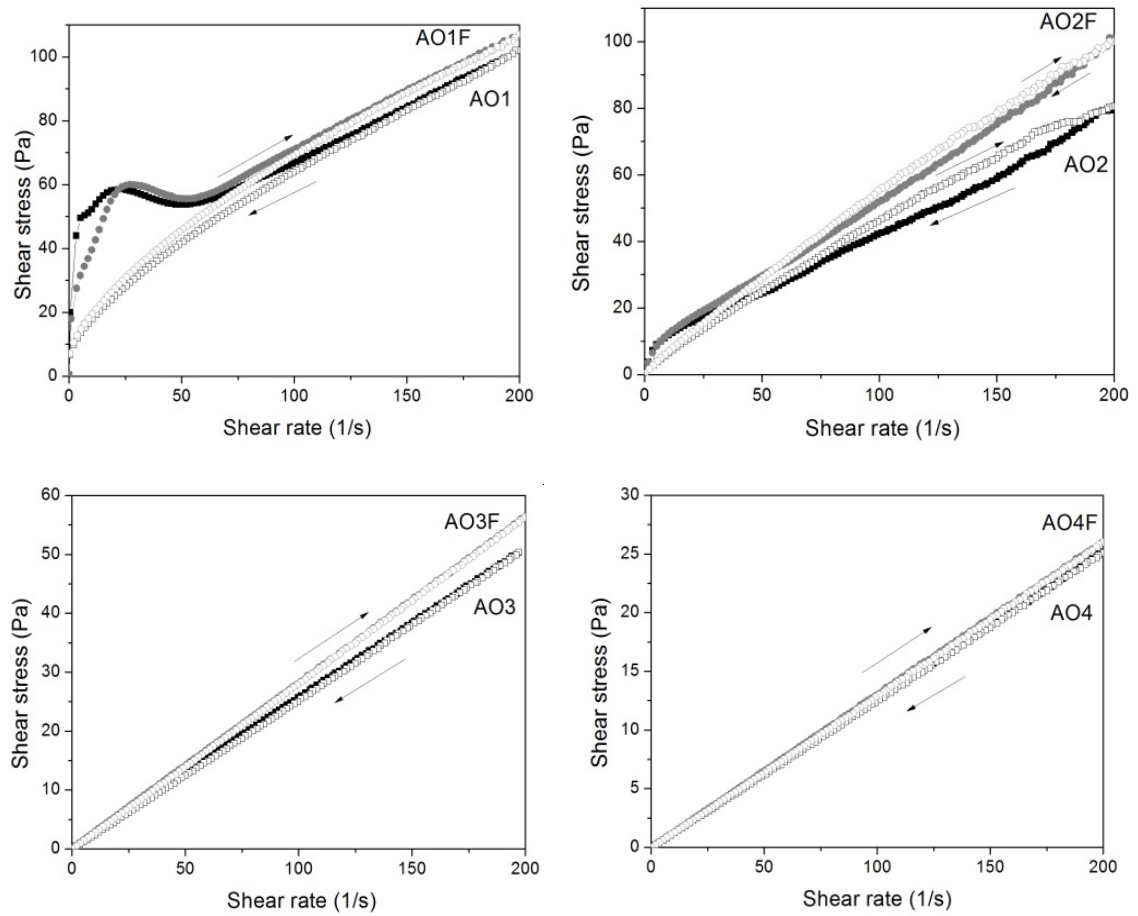

Figure 4: Continuous shear rheometry assay of fluconazole-loaded (grey symbols) and unloaded formulations AO1-AO4 (black symbols). The closed symbols represent upward flow curves, and the open symbols represent downward flow curves.

The oscillatory rheological data are displayed in Figure 5 as the storage modulus (G') and loss modulus (G') as a function of the oscillatory frequency. The storage modulus is a measure of the energy stored and recovered per deformation cycle and represents the solidlike component of a viscoelastic material. The storage modulus is large when a sample is predominantly elastic or highly structured. The loss modulus is a measure of the energy dissipated per cycle and represents the liquid-like component [18]. The frequency sweep analyses suggest that $\mathrm{AO} 3-\mathrm{AO} 4$ are more viscous and less elastic at the selected frequency because G" > G' and because both moduli are frequency dependent. For AO1-AO2, the G' values were high and relatively independent of frequency. The slopes of the lamellar phase formulations (AO1-AO2) 

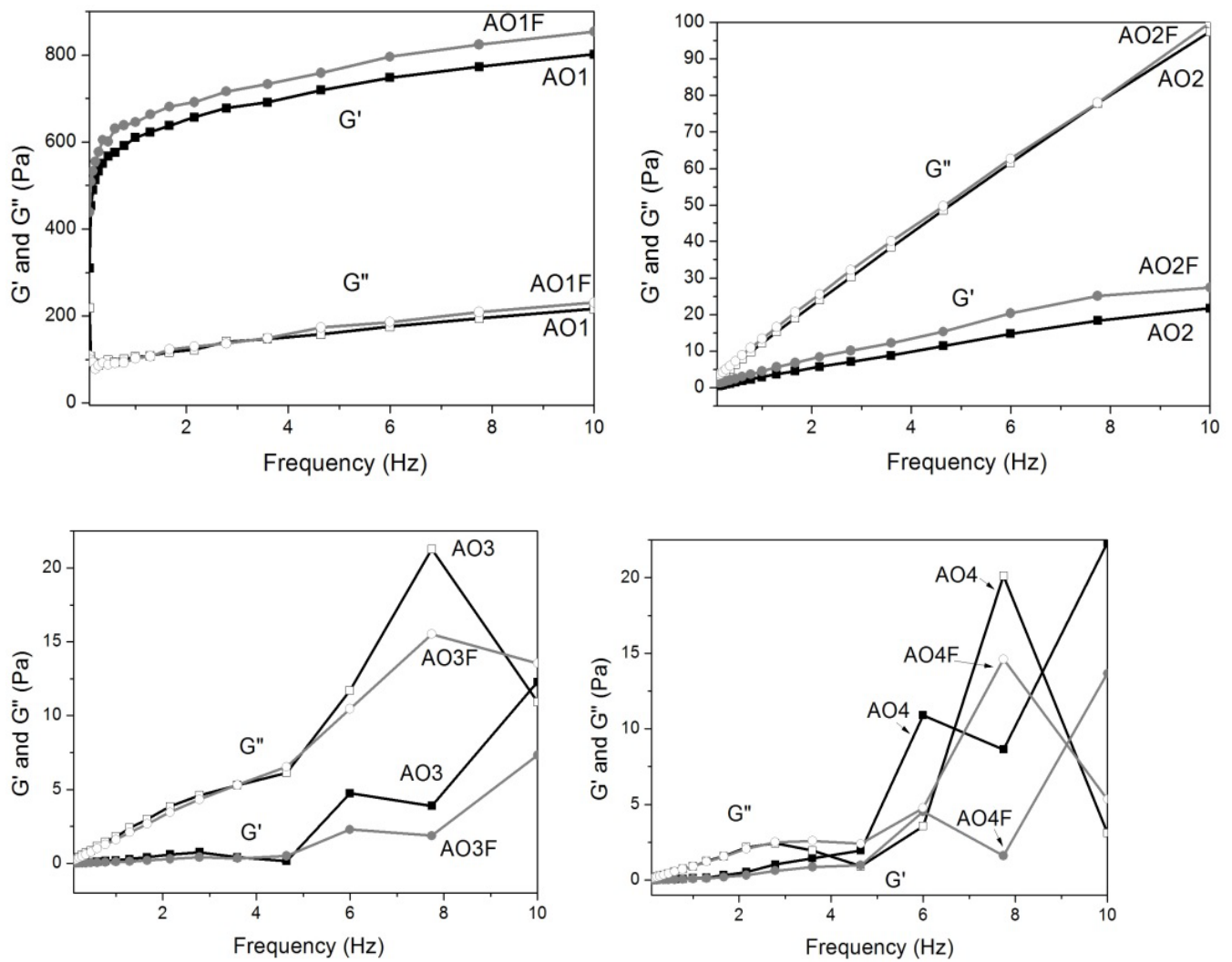

Figure 5: Frequency sweep profile of the storage modulus (G' - closed symbols) and loss modulus (G" - opened symbols) of fluconazole-loaded (grey symbols) and unloaded formulations $\mathrm{AO} 1-\mathrm{AO} 4$ (black symbols) at $25^{\circ} \mathrm{C}$.

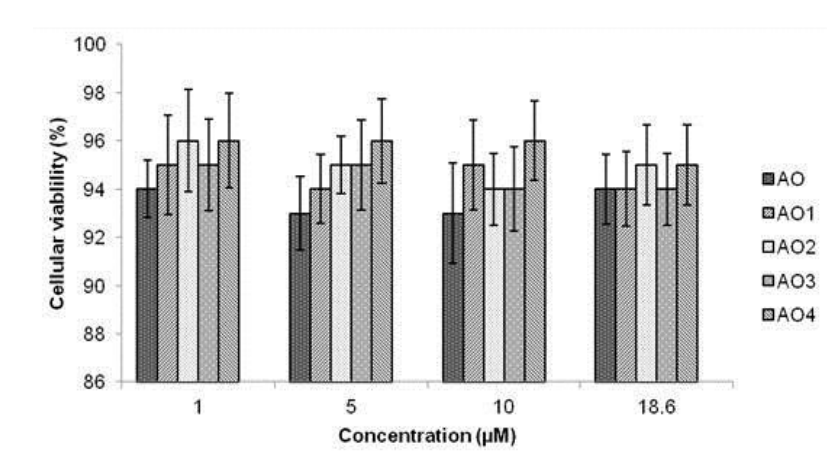

Figure 6: Cellular viability of unloaded formulations ( $\mathrm{AO} 1, \mathrm{AO} 2, \mathrm{AO} 3, \mathrm{AO} 4)$ and oleic acid $(\mathrm{AO})$

were lower than the slopes of the liquid isotropic systems AO3-AO4, indicating a more structured character for the lamellar phases.

\section{Safety evaluation of the formulations}

In vitro unspecific cytotoxicity: In vitro unspecific cytotoxicity studies of the formulations were performed using J-774 mouse macrophages as the cellular model. The data (Figures 6 and 7) were exhibited in per cent of cellular viability. The assay results of cellular viability showed that the unloaded samples $(\mathrm{AO}, \mathrm{AO} 1, \mathrm{AO} 2, \mathrm{AO}$, $\mathrm{AO} 4)$ and loaded samples (AOF, AO1F, AO2F, AO3F, AO4F) were unable to kill normal cell macrophages. All samples exhibited cellular viability greater than that $92 \%$, and therefore, it is suggested that the formulations and the drug do not present toxic activity or cytotoxicity.

\section{In vivo evaluation:}

Histomorphometric analysis of the epidermis and dermis: The results for the thickness of the epidermis and dermis are presented in Table 2. Analysis of variance (ANOVA) verified a normal distribution of data, and the experiment followed a completely randomised design. The $\mathrm{F}$ test was used to detect differences between treatments. The means of groups were compared, two by two, by the Tukey test.

The data suggest that the groups AO, PPG-5-CETETH-20, and formulation $\mathrm{AO} 3 \mathrm{~F}$ caused significantly higher thickening of the epidermis and dermis than the control (Figure 8). This result suggests an increase of skin hydration that would be revealed by an increase in the interstitial spaces. It is known that some cosmetic formulations, with or without active substances, may promote skin hydration, to the benefit of the skin [19].

According to Libardi [20], what makes the skin stay healthy and soft, with good flexibility and elasticity is the maintenance of skin hydration and the ability of the organism not only to stimulate cell renewal but also to synthesise components of the epidermis. For good functioning of the mechanism of skin hydration, the stratum corneum should be able to retain water so that its rate of evaporation is limited to a normal level. Proksch et al. [21] claim that skin hydration is intrinsically related to the integrity of the stratum corneum.

The properties of occlusion and moisturising of the components were observed for the formulation $\mathrm{AO} 3 \mathrm{~F}$, characterised as a micellar system. The occlusive activity conferred by nano-systems has been cited in other works such as Brinon et al. [22] Those authors suggest that this 


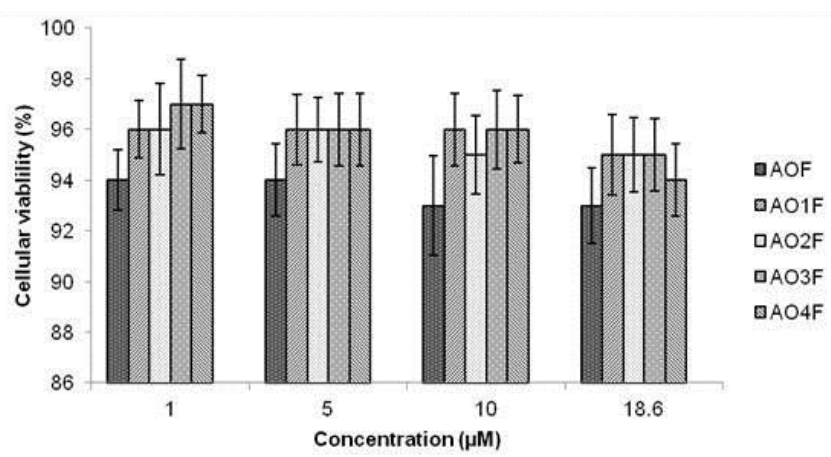

Figure 7: Per cent cellular viability of fluconazole-loaded formulations (AO1F, AO2F, AO3F, AO4F) and loaded oleic acid (AOF).

\begin{tabular}{|c|c|c|c|c|c|c|c|c|}
\hline \multirow{2}{*}{ Group } & \multicolumn{2}{|c|}{ Epidermis $(\boldsymbol{\mu m})$} & \multicolumn{2}{|c|}{ Dermis $(\boldsymbol{\mu m})$} & \multicolumn{2}{c|}{ Number of fibroblasts } & \multicolumn{2}{c|}{ Number of leukocytes } \\
\cline { 2 - 9 } & Average & SD & Average & SD & Average & SD & Average & SD \\
\hline S & 32.92 & 1.66 & 1136.76 & 37.20 & 12.47 & 2.30 & 2.6 & 1.65 \\
\hline AO & 35.56 & 5.79 & 1097.83 & 47.64 & 12.27 & 2.30 & 1.87 & 0.27 \\
\hline AO3F & 30.24 & 3.63 & 1014.82 & 66.79 & 12.67 & 2.59 & 0.27 & 0.10 \\
\hline C+ & 37.58 & 3.00 & 1269.11 & 53.04 & 7.83 & 1.68 & 6.10 & 3.00 \\
\hline C- & 23.95 & 1.62 & 672.95 & 51.48 & 9.10 & 1.65 & 0.67 & 0.88 \\
\hline
\end{tabular}

Table 2: Thickness of the epidermis and dermis $(\mu \mathrm{m})$. Number of fibroblasts and leukocytes in $250 \mu \mathrm{m}^{2}$ of the papillary dermis in each experimental group. Key: (S) PPG5-CETETH-20, (AO) oleic acid, (AO3F) fluconazole-loaded tested formulation, $\left(\mathrm{C}^{+}\right)$positive control, (C-) negative control, (SD) standard deviation.

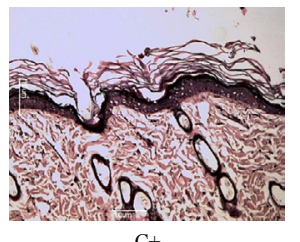

$\mathrm{C}+$

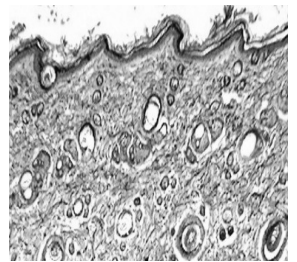

10
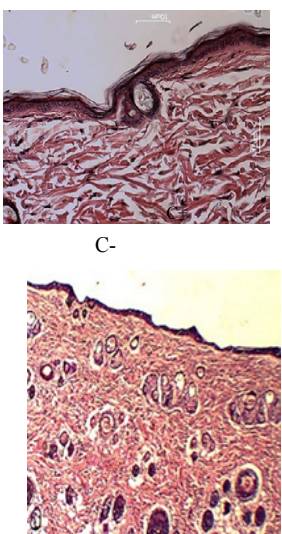

$\mathrm{AO3F}$

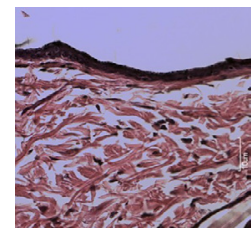

Figure 8: Photomicrographs of sections of the epidermis and dermis
oleic acid; AO3F: sample; C+: positive control; C-: negative control.

action is related to the characteristic colloidal structure of the emulsified system, in which the organisation of the surfactant interfacial layer is similar to that of the lipid bilayers that join keratinocytes, increasing cohesion and, consequently, the ability to form a continuous film [23].

Histopathological analysis of the dermis: The presence of fibroblasts and leukocytes in larger amounts during skin irritation can be used to predict irritation. The number of fibroblasts and leukocytes counted in the papillary dermis of the various experimental groups is given in Table 2. By ANOVA, the distribution of the data for the number of fibroblasts and leukocytes per area was found to be non-normal, so the Kruskal-Wallis test $(\mathrm{p}<0.05)$ was used to analyse the results.

The most common adverse reactions of topical formulations is skin irritation, which can be seen as local intolerance and may elicit minor discomfort, as well as acute reactions that vary in intensity from burning to itching. This irritation may lead to corrosion and tissue destruction. All of these reactions are restricted to the area in direct contact with the product. Generally, the degree of irritation is related to the concentration of an ingredient in the final product and the cosmetic formulation [24].

It can be said that irritation causes a skin condition similar to that produced by a lesion. Lesions in skin tissue are characterised as any events that impair the structure or function of the tissue. They produce changes in the ability of cells to perform their normal homeostatic functions, and they trigger various histochemical and biological processes that lead to vasomotor action to mobilise all the cells responsible for fighting infection and/or the offending agent. These processes are intended to replace losses of substances as quickly as possible and to restore the physiological balance and contribute to the survival of the organism [25]. 


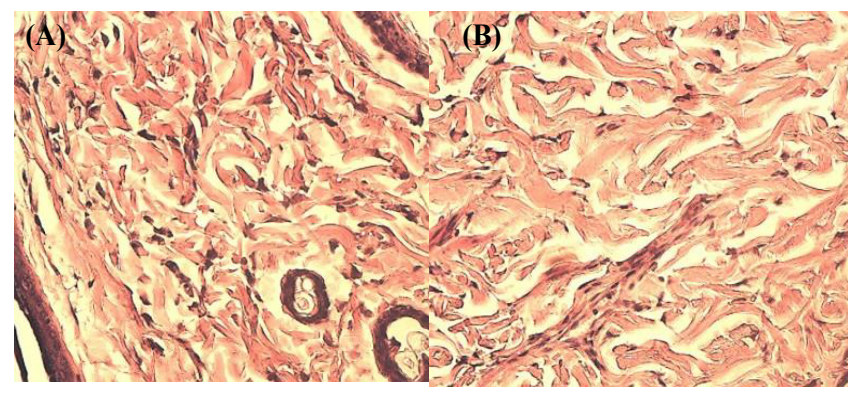

Figure 9: Photomicrographs showing the papillary dermis in areas: $(A) C-$; (B) picture representative of the treatments with $\mathrm{S}, \mathrm{AO}, \mathrm{AO} 3 \mathrm{~F}$ and $\mathrm{C}+$, where a greater number of fibroblasts are seen in the dermis $(1000 \mathrm{x})$.

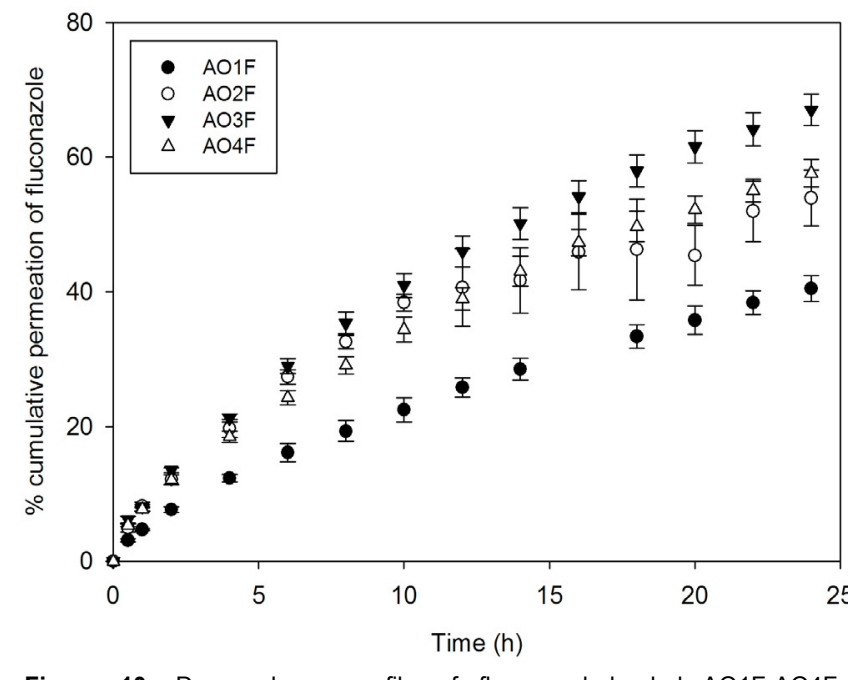

Figure 10: Drug release profile of fluconazole-loaded AO1F-AO4F formulations, $10 \mathrm{mg} / \mathrm{mL}(\mathrm{n}=6)$.

At an early stage, an irritation process releases inflammatory mediators and mobilises a sequence of cells, especially neutrophils and lymphocytes, to combat the offending agent or to start the immune processes that facilitate healing [26]. Inflammatory mediators act as chemotactic signals and growth factors for the inflammatory cells, which are represented by polymorphonuclear leukocytes (PMNs), macrophages, and lymphocytes. These cells also release growth factors for endothelial cells, fibroblasts and keratinocytes. The release of all of these factors can lead to the formation of granulation tissue [27].

Although the results of the leukocyte count of PPG-5-CETETH-20 and oleic acid were higher than those obtained for the control, these counts still remain low. Importantly, these results may have occurred through the use of pure raw materials in the animal's skin.

The results obtained for the formulation AO3F were not significantly different from those obtained for the control group, suggesting no occurrence of inflammatory processes when administering a formulation containing $40 \%$ oil, $40 \%$ surfactant and $20 \%$ water.

Other promising data are related to the count of fibroblasts. It was observed that the number of fibroblasts was significantly higher for the various experimental groups than for the control (Figure 9). This result indicates that the formulations may also improve properties related to skin support and cell regeneration because these cells will originate collagen and elastin fibres.

The fibroblast is the main cell involved in the formation of granulation tissue, synthesising hyaluronic acid, fibronectin, collagens type I and III, elastin and proteases, such as collagenase [25]. The formulation $\mathrm{AO} 3 \mathrm{~F}$ increased the production of fibroblasts. This, in turn, increased the production of collagen, which is responsible for the strength and resistance of greater thickness and dermal trauma, promoting satisfactory skin restructuring $[28,29]$.

\section{Proof of concept of the formulations}

The drug release assay enables in vitro analysis of drug movement across a membrane using a two-compartment model. The donor compartment contains the formulation, and a non-rate-limiting membrane separates the compartments and supports the dose. The amount of fluconazole released was expressed as a percentage, which was plotted against time in Figure 10. In addition, the pseudo-steadystate flux $(\mathrm{J}, \mu \mathrm{g} / \mathrm{min} \mathrm{cm})$ derived from Fick's law was calculated as the gradient of the linear portion of the drug release curve [30]. The results are listed in Table 3.

This study revealed that $\mathrm{AO} 3 \mathrm{~F}-\mathrm{AO} 4 \mathrm{~F}$ released more fluconazole than AO1F-AO2F. This phenomenon most likely occurs because the latter formulations present a liquid crystalline network structure with higher elasticity than that of AO3F-AO4F. The less ordered AO3F-AO4F systems with reduced viscosity allowed a higher degree of mobility of drug molecules in their matrices.

The OA sample showed the highest value of flux (J), followed by AO3F. The other formulations did not present a significant difference. Because the AO sample presents no structural organisation, such as the liquid crystalline samples or the micellar system, the drug was released faster. The flux of AO3F could have been higher due to its position in the phase diagram of Figure 1. This sample was placed in a phase transition area, and therefore, its structure may not control drug release as much as the $\mathrm{AO} 1 \mathrm{~F}, \mathrm{AO} 2 \mathrm{~F}$ or $\mathrm{AO} 4 \mathrm{~F}$ systems. $\mathrm{AO} 1 \mathrm{~F}$ was shown to prolong drug release to the greatest extent. This result may be due to the highest elastic modulus in AO1F, which increases the stiffness of the liquid crystalline matrix. In addition to the differences between the drug release rates, all formulations released fluconazole in a controlled manner because an exponential pattern governed drug diffusion, as seen in the curves of Figure 10. Therefore, this study showed that structural modifications of surfactant systems can modify the rate and profile of drug delivery.

Permeation studies were performed in Franz diffusion cells using the skin of porcine ear. The cumulative permeation of fluconazole incorporated into formulations $\mathrm{AO} 1 \mathrm{~F}-\mathrm{AO} 4 \mathrm{~F}$ was calculated for $6 \mathrm{~h}$ and $12 \mathrm{~h}$ of experiment, as was the drug retention in the stratum corneum, dermis, and epidermis. The values obtained are listed in Table 3.

The sample AOF promoted the highest drug permeation, followed by $\mathrm{AO} 4 \mathrm{~F}$, which contains the largest quantity of oleic acid among the other formulations. This result indicates the enhancing effect of oleic acid on the in vitro permeability of fluconazole. Oleic acid may reduce the lipid packing order in epithelial cell membranes. This effect could increase the fluidity of the lipid layer and decrease the diffusion resistance, thus assisting the permeation of compounds through the skin [31]. Therefore, AO4F may be explored for a systemic effect because it promoted drug transportation across the skin. This suggests that the drug may reach the blood circulation. 
Citation: Silva HR, Luz GM, Satake CY, Correa BC, Sarmento VHV, et al. (2014) Surfactant-based Transdermal System for Fluconazole Skin Delivery. J Nanomed Nanotechnol 5: 231. doi: 10.4172/2157-7439.1000231

Page 9 of 10

\begin{tabular}{|c|c|c|c|c|c|c|c|c|}
\hline & \multirow{2}{*}{\multicolumn{2}{|c|}{ Drug release }} & \multirow{2}{*}{\multicolumn{2}{|c|}{ Drug permeation $\left(\mu \mathrm{g} . \mathrm{cm}^{2}\right)$}} & \multicolumn{4}{|c|}{ Skin retention $\left(\mu \mathrm{g} . \mathrm{cm}^{2}\right)$} \\
\hline & & & & & \multicolumn{2}{|c|}{ Stratum corneum } & \multicolumn{2}{|c|}{ Epidermis+dermis } \\
\hline & $J\left(\mu \mathrm{g} / \mathrm{cm}^{2}\right)$ & $\mathbf{r}^{2}$ & $6 \mathrm{~h}$ & $12 \mathrm{~h}$ & $6 \mathrm{~h}$ & $12 \mathrm{~h}$ & $6 \mathrm{~h}$ & $12 \mathrm{~h}$ \\
\hline AOF & 132.12 & 0.98 & $319.2 \pm 37.2$ & $673.6 \pm 10.6$ & $2.87 \pm 0.31$ & $9.79 \pm 2.33$ & $19.87 \pm 8.39$ & $13.43 \pm 1.58$ \\
\hline $\mathrm{AO} 1 \mathrm{~F}$ & 52.39 & 0.97 & 0 & 0 & $0.61 \pm 0.32$ & $11.38 \pm 2.56$ & $18.58 \pm 3.55$ & $7.19 \pm 1.52$ \\
\hline $\mathrm{AO} 2 \mathrm{~F}$ & 59.89 & 0.99 & 0 & 0 & $2.35 \pm 0.12$ & $2.15 \pm 0.43$ & $5.37 \pm 0.41$ & $6.96 \pm 2.08$ \\
\hline $\mathrm{AO} 3 \mathrm{~F}$ & 71.09 & 0.98 & $0.4 \pm 0.1$ & $4.3 \pm 1.4$ & $2.44 \pm 0.54$ & $0.10 \pm 0.4$ & $5.42 \pm 1.09$ & $2.96 \pm 1.14$ \\
\hline $\mathrm{AO} 4 \mathrm{~F}$ & 53.38 & 0.99 & $85.9 \pm 28.4$ & $130.3 \pm 5.8$ & $1.02 \pm 0.37$ & $9.60 \pm 3.03$ & $17.59 \pm 1.23$ & $7.59 \pm 1.19$ \\
\hline
\end{tabular}

Table 3: Flux $(\mathrm{J})$ and correlation coefficient $\left(\mathrm{r}^{2}\right)$ derived from drug release curves, drug permeated through the skin at $6 \mathrm{~h}$ and $12 \mathrm{~h}$, and drug retained in the stratum corneum and in the epidermis + dermis at $6 \mathrm{~h}$ and $12 \mathrm{~h}$. Data were collected for fluconazole-loaded formulations (AO1F, AO2F, AO3F, AO4F) and loaded oleic acid (AOF), all containing $10 \mathrm{mg} / \mathrm{mL}$ of drug. The values represent the average \pm standard deviation of six replicates.

In contrast, even though $\mathrm{AO} 1-\mathrm{AO} 3$ had retained more drug in the epidermis plus dermis than in the stratum corneum barrier, there was no drug permeation for these systems, which leads us to conclude that these samples promoted skin retention. In comparing AO1F-AO3F, an increase in the drug concentration in the stratum corneum with time was observed. $\mathrm{AO} 1 \mathrm{~F}$ and $\mathrm{AO} 2 \mathrm{~F}$ are lamellar phases, but $\mathrm{AO} 1 \mathrm{~F}$ exhibited a higher elastic modulus, which is characteristic of a more structurally organised system. Therefore, it can be concluded that the higher organisation of the liquid crystalline structure of AO1F contributed to controlling drug release.

It is reported that the concentration of antifungal drug attained in the skin is an important factor in the treatment of dermatomycosis and that the presence of the therapeutically active form in the skin is closely related to the efficacy of the drug. However, this evidence was found for oral treatments [9]. Considering the topical application of the formulations studied here, it is possible to promote drug permeation or skin retention by changing only the composition of the components in the formulations. These results open the way to considering PPG5-CETETH-20-based transdermal systems for antifungal skin delivery for Sporotrichosis treatment.

\section{Conclusions}

The findings of this work noted the great potential of PPG-5CETETH-20-based transdermal systems for either systemic or local action because it was found that both formulations enhanced drug permeation and skin retention. In vitro and in vivo biological assays showed that the formulations did not affect normal cell macrophages and did not promote skin irritation. Therefore, the results presented here provide new possibilities for transdermal systems with different structural and rheological characteristics for the treatment of Sporotrichosis using antifungal drugs.

\section{Acknowledgment}

The authors thank the LNLS (The Brazilian Synchrotron Light Laboratory) for SAXS measurements and FAPESP (São Paulo Research Foundation), CNPq (National Council for Scientific and Technological Development) and PADC-UNESP (Programa de Apoio ao Desenvolvimento Científico) for the financial support.

\section{References}

1. Bonifaz A, Saúl A, Paredes-Solis V, Fierro L, Rosales A, et al. (2007) Sporotrichosis in childhood: clinical and therapeutic experience in 25 patients. Pediatr Dermatol 24: 369-372.

2. Vásquez-del-Mercado E, Arenas R, Padilla-Desgarenes C (2012) Sporotrichosis. Clin Dermatol 30: 437-443.

3. De Araujo T, Marques AC, Kerdel F (2001) Sporotrichosis. Int J Dermatol 40: 737-742.

4. Yang F, Kamiya N, Goto M (2012) Transdermal delivery of the anti-rheumatic agent methotrexate using a solid-in-oil nanocarrier. Eur J Pharm Biopharm 82 158-163.
5. Naik A, Kalia YN, Guy RH (2000) Transdermal drug delivery: overcoming the skin's barrier function. Pharm Sci Technolo Today 3: 318-326.

6. Carvalho FC, Rocha e Silva H, da Luz GM, Barbi Mda S, Landgraf DS, et al. (2012) Rheological, mechanical and adhesive properties of surfactantcontaining systems designed as a potential platform for topical drug delivery. $J$ Biomed Nanotechnol 8: 280-289.

7. Carvalho FC, Campos ML, Peccinini RG, Gremião MP (2013) Nasa administration of liquid crystal precursor mucoadhesive vehicle as an alternative antiretroviral therapy. Eur J Pharm Biopharm 84: 219-227.

8. Bruschi ML, Freitas O, Lara EHG, Panzeri H, Gremião MPD, et al. (2008) Precursor system of liquid crystalline phase containing propolis microparticles for the treatment of periodontal disease: development and characterization. Drug Develop Ind Pharm 34: 267-278.

9. Sobue S, Sekiguchi K, Nabeshima T (2004) Intracutaneous distributions of fluconazole, itraconazole, and griseofulvin in Guinea pigs and binding to human stratum corneum. Antimicrob Agents Chemother 48: 216-223.

10. Olfert ED, Cross BM, McWilliam AA (1993) Guide to the care and use of experimental animals. Canadian Council on Animal Care, Ottawa, Canada.

11. Polacow MLO, Pires-de-Campos MSM, Leonardi GR, Carvalho LS, Ribeiro MCAP, et al. (2004) Eleito do ultra-som na permeação cutânea do tiratricol: análise histológica. Rev Bras Fisioter 8: 53-60.

12. Dick IP, Scott RC (1992) Pig ear skin as an in-vitro model for human skin permeability. J Pharm Pharmacol 44: 640-645.

13. Hosmer J, Reed R, Bentley MVLB, Nornoo A, Lopes LB (2009) Microemulsions containing medium-chain glycerides as transdermal delivery systems for hydrophilic and hydrophobic drugs. AAPS Pharm Sci Tech 10: 589-596.

14. Herai H, Gratieri T, Thomazine JA, Bentley MV, Lopez RF (2007) Doxorubicin skin penetration from monoolein-containing propylene glycol formulations. Int J Pharm 329: 88-93.

15. Lopez RF, Bentley MV, Begoña Delgado-Charro M, Guy RH (2003) Optimization of aminolevulinic acid delivery by iontophoresis. J Control Release 88: 65-70.

16. Holmqvist $P$, Alexandridis $P$, Lindman $B$ (1997) Modification of the microstructure in poloxamer block copolymer-water-"oil" systems by varying the "oil" type. Macromolecules 30: 6788-6797.

17. Fanun M (2008) A study of the properties of mixed nonionic surfactants microemulsions by NMR, SAXS, viscosity and conductivity. J Mol Liquids 142 103-110.

18. Barnes HA, Hutton JF, Walter K (1989) An introduction to rheology. Elsevier, New York, USA.

19. Leonardi GR, Gaspar LR, Maia Campos PM (2002) Application of a noninvasive method to study the moisturizing effect of formulations containing vitamins A or E or ceramide on human skin. J Cosmet Sci 53: 263-268.

20. Libardi FS (1998) Lactato de amônio. Cosmet Toiletries 11: 50-53.

21. Proksch E, Nissen HP, Bremgartner M, Urquhart C (2005) Bathing in a magnesium-rich Dead Sea salt solution improves skin barrier function, enhances skin hydration, and reduces inflammation in atopic dry skin. Int $J$ Dermatol 44: 151-157.

22. Brinon L, Geiger S, Alard V, Doucet J, Tranchant JF, et al. (1999) Percutaneous absorption of sunscreens from liquid crystalline phases. J Control Release 60: 67-76.

23. Klein K (2002) Liquid crystals and emulsions: a wonderful marriage. Cosmet Toiletries 117: 30-34. 
Citation: Silva HR, Luz GM, Satake CY, Correa BC, Sarmento VHV, et al. (2014) Surfactant-based Transdermal System for Fluconazole Skin Delivery. J Nanomed Nanotechnol 5: 231. doi: 10.4172/2157-7439.1000231

Page 10 of 10

24. http://portal.anvisa.gov.br/wps/portal/anvisa/home

25. Kitchen S, Young S (1998) Reparo dos tecidos. In: Kitchen S, Bazin S. Eletroterapia de.

26. Andrews JM (1995) Cirurgia plástica, Atheneu, São Paulo.

27. http://www2.fcfar.unesp.br/Home/Pos-graduacao/CienciasFarmaceuticas/ MarlusChorilliDO.pdf

28. Chorilli M, Prestes PS, Rigon RB, Leonardi GR, Chiavacci LA, et al. (2011) Structural characterization and in vivo evaluation of retinyl palmitate in nonionic lamellar liquid crystalline system. Surf B Biointerfaces 85: 182-188.
29. Shapiro SS, Saliou C (2001) Role of vitamins in skin care. Nutrition 17: 839844

30. Williams AC (2003) Transdermal and topical drug delivery. Pharmceutical Press, London, UK.

31. Nicolazzo JA, Reed BL, Finnin BC (2005) Buccal penetration enhancers--how do they really work? J Control Release 105: 1-15. 